\title{
Editorial: Simulation and Modeling of Multiphase Transport in Industrial Process and Facility
}

\author{
Nan Gui * \\ Institute of Nuclear and New Energy Technology, Tsinghua University, Beijing, China
}

Keywords: high temperature gas-cooled reactor, marine reactor, liquefied petroleum gas plant, pebble bed, generation-IV reactor

Editorial on the Research Topic

Simulation and Modeling of Multiphase Transport in Industrial Process and Facility

Facing the dual pressure of environmental problems and energy issues, much effort has been made to find new energy alternatives like nuclear energy or upgrade the existing technologies such as petrochemical technology.

To utilize the nuclear energy, many types of nuclear reactors have been developed worldwide after decades of research, among which the pebble bed high temperature gas-cooled reactor (pebble bed HTGR) has attracted wide attention and been regarded as a promising generation-IV reactor. It uses large fuel pebbles and helium gas as coolant. Inside the core, the pebble bed flow, a kind of extremely slow flow of pebbles, plays a critical role for the reactor core design and safety analysis. To gain a better understanding of the flow characteristics, we focus on various fundamental issues associated with pebble bed flow, such as the quasi-static packing structure, the flow velocity, the flow pattern, the resident time, and stagnant ratio, etc. In the work (Huang et al.), discharging characteristics of particle clusters with different entangle angles in particle bed-type of HTGR is investigated through discrete element method (DEM) modeling. The effects of entanglement angle and aspect ratio on the characteristics of particle flow are studied in this paper.

Apart from land-based nuclear reactors, nuclear reactors are also used for propulsion power sources for military nuclear power ships, icebreakers, floating platforms, and deep-sea probes. Operating in a dramatically changing marine environment, these marine reactors are subjected to additional motions such as heeling, heaving, rolling, and pitching generated by ocean waves. The ocean conditions will inevitably affect the flow field inside the reactor and result in large fluctuation of flow rate. From the perspective of reactor safety, the effect of oscillating motion on the flow characteristics is explored through computational fluid dynamics (CFD) in the work (Gong and Wu). Based on a full-scale three-dimensional oscillating marine reactor, the flow distribution, velocity profile as well as pressure distribution under different oscillating angle and elevation are presented, which is useful for understanding the fluid flow in marine reactors under the ocean environment.

In addition, with the development of petrochemical industry, the liquefied petroleum gas (LPG), a relatively clean and a supreme source of energy, is also being used as a key component in the global energy supply. The international trade agreements, the chemical and non-chemical demand of liquefied petroleum gas with the increase in the world's population have brought its production to the limelight. During processing, a variety of components are extracted, including methane and ethane, which exist in large quantities as natural gas. In the work (Ali et al.), with the focus of saving processing cost and energy, the LPG recovery performance is studied by the design and simulation of a liquefied petroleum gas plant using Aspen HYSYS. To obtain the maximum yield of LPG from natural gas with increased purity, different schemes of process alternatives are applied by reducing 
the sizes of the exchanger and other pieces of equipment. This investigation can benefit the energy optimization and cost optimization for the recovery of LPG from natural gas.

Overall, the past decades have seen great progress in these new technologies. Still, the fundamental issues including the mechanism of pebble bed flow, fluid flow and heat transfer in reactor, and the improvement of existing petrochemical technology need to be further investigated.

\section{AUTHOR CONTRIBUTIONS}

The author confirms being the sole contributor of this work and has approved it for publication.
Conflict of Interest: The author declares that the research was conducted in the absence of any commercial or financial relationships that could be construed as a potential conflict of interest.

Publisher's Note: All claims expressed in this article are solely those of the authors and do not necessarily represent those of their affiliated organizations, or those of the publisher, the editors and the reviewers. Any product that may be evaluated in this article, or claim that may be made by its manufacturer, is not guaranteed or endorsed by the publisher.

Copyright (c) 2022 Gui. This is an open-access article distributed under the terms of the Creative Commons Attribution License (CC BY). The use, distribution or reproduction in other forums is permitted, provided the original author(s) and the copyright owner(s) are credited and that the original publication in this journal is cited, in accordance with accepted academic practice. No use, distribution or reproduction is permitted which does not comply with these terms. 